\title{
BMJ open Semen quality and reproductive hormones in Faroese men: a cross-sectional population-based
study of 481 men
} Jónrit Halling, ${ }^{1}$ Maria Skaalum Petersen, ${ }^{1}$ Niels Jørgensen, ${ }^{2}$ Tina Kold Jensen, ${ }^{2,3}$ Philippe Grandjean, ${ }^{3,4}$ Pál Weihe ${ }^{1}$

To cite: Halling J, Petersen MS, Jørgensen N, et al. Semen quality and reproductive hormones in Faroese men:

a cross-sectional populationbased study of 481 men. BMJ Open 2013;3:e001946. doi:10.1136/bmjopen-2012001946

- Prepublication history and additional material for this paper are available online. To view these files please visit the journal online (http://dx.doi.org/10.1136/ bmjopen-2012-001946).

The first two authors should be regarded as joint First Authors.

Received 9 August 2012 Revised 4 February 2013 Accepted 6 February 2013

This final article is available for use under the terms of the Creative Commons Attribution Non-Commercial 2.0 Licence; see http://bmjopen.bmj.com

For numbered affiliations see end of article.

\footnotetext{
Correspondence to Jónrit Halling; jonrit@health.fo and Maria Skaalum Petersen; maria@health.fo
}

\begin{abstract}
Objectives: To determine semen quality and reproductive hormone levels in young Faroese men.

Design: Descriptive cross-sectional study of Faroese men compared with Danish men.

Setting: Faroese one-centre study.

Participants: 481 men born from 1981 to 1987 and investigated from 2007 to 2010.
\end{abstract}

Outcome measures: Sperm concentration, semen volume, total sperm count, sperm motility, sperm morphology and reproductive hormone levels.

Results: Sperm concentrations for the Faroese men were lower than for the Danish men (crude median 40 vs $48 \mathrm{mill} / \mathrm{ml}, \mathrm{p}<0.0005)$. Semen volume was higher, and thus the total sperm counts did not differ (159 vs 151 mill, $p=0.2$ ). Motility and morphology did not differ between the Faroese and Danes. The inhibin B/follicle-stimulating hormone ratios for the Faroese men were lower than for the Danes (64 vs 76 , $\mathrm{p}=0.001)$. Similarly, lower total testosterone/luteinising hormone $(\mathrm{LH})$ ratio (4.6 vs $6.0, p<0.0005)$ and lower calculated free-testosterone/LH ratio (94 vs 134, $p<0.0005)$ were detected for the Faroese men.

Conclusions: Semen quality among the Faroese men is at the same low level as reported for Danish men, and the reproductive hormone levels furthermore indicated a lower Leydig cell capacity for testosterone production. The influence of environmental exposure and genetic factors on semen quality has to be studied further.

\section{INTRODUCTION}

In 1992, Carlsen et al ${ }^{1}$ published a combined analysis of results from 61 papers published between 1939 and 1991 and showed a significant decline in sperm counts over a 50-year period. A detailed reanalysis of the results found that the conclusion was supported by the underlying studies. ${ }^{2}{ }^{3}$ Following the 1992 publication, many analysed retrospectively their historical data for temporal trends,

\section{ARTICLE SUMMARY}

Article focus

- Semen quality studies have not been conducted in the Faroe Islands before.

- Owing to the Faroese population differing from other populations regarding, for example, high exposure to persistent organic pollutants (POPs) and genetic diseases, the semen quality of the Faroese men from the general population would be expected to be low.

Key messages

- The semen quality among Faroese men is at the same low level as reported in Denmark.

- The low inhibin B/follicle-stimulating hormone ratio for the Faroese men corroborates the finding of low sperm counts and provides independent evidence of poorer testicular function in the Faroese men than in the Danes.

- Similarly, the Leydig cell capacity for testosterone production was also lower for the Faroese men than for the Danes.

Strengths and limitations of this study

- Prospectively designed, cross-sectional study of testicular function among young men from the general population unselected with regard to fertility.

- Standardised inclusion and investigation procedures.

- Clinical examination for one subgroup of Faroese men, but not for the other.

some finding a decline and others not. Realising that the trend analyses indicated that semen quality could have reached a low level where it might affect fecundity, several prospectively designed cross-sectional semen quality studies were initiated to determine the current quality in men from general populations. These studies not only revealed geographical differences ${ }^{4} 5$ but also confirmed the general presence of low semen 
quality in men from all investigated countries when the results were interpreted according to available publications focused on associations between semen quality and fertility chances. ${ }^{6-9}$

The causes of decreased semen quality are not clear, but it is feasible that many cases may have been caused by exposure to environmental factors in utero, during adolescence or in adulthood; ${ }^{10}$ however, most likely acting on a background of different genetic susceptibility.

The Faroese population differs from other populations in many respects. The Faroese population has high exposure to persistent organic pollutants (POPs) derived from traditional marine food, which includes blubber from the pilot whale. At the same time, there are several genetic diseases reported with very high frequency in the Faroese Islands. ${ }^{11}$ Thus, our hypothesis was that the semen quality of Faroese men from the general population would be quite low.

\section{MATERIALS AND METHODS}

To test our hypothesis, we investigated the semen quality of young Faroese men from the general population in 2007-2010. For comparison, we used a recently published similar group of Danish men examined in 2006-2010 ${ }^{12}$ and the current WHO reference levels. ${ }^{13}$

\section{Study population: men from the general Faroese population}

The entire study population consisted of 484 men, originating from two separate studies. The first study group included 241 randomly selected young men examined between February 2007 and February 2009 (F1). The second group comprised 243 men examined between November 2009 and November 2010 (F2). A detailed description of the study population based on questionnaire information and results from the physical examination (see below) is summarised in table 1. Within 3 months prior to participation, 132 men $(27.4 \%)$ had used medication, mainly antibiotics, painkillers or asthma/allergy medicine.

\section{Faroese men, examined 2007-2009 (subgroup F1)}

Invitation letters to participate in the study were sent to 1100 men, consecutively listed in the Faroese population register as born between January 1981 and December 1984 , followed by a phone call to arrange the examination details. A total of 34 men had emigrated, and 43 letters were returned as undeliverable. Thus, 1023 were invited. Of these, 490 could not be reached and 292 declined to participate. Hence, the final F1 group comprised 241 men ( $24 \%$ of all invited).

\section{Faroese men, examined 2009-2010 (subgroup F2)}

This group consisted of a cohort generated from consecutive births at the three Faroese hospitals during 1986-1987 (N=509 men) as described elsewhere. ${ }^{14-16}$ Detailed information on their physical health and potential environmental factors was collected at the time of birth and during the course of follow-up at ages 7, 14 and 22 years. The 421 men who had participated in the 22-year follow-up were invited to participate in the semen quality study. This was the sole selection criterion. All men received a letter of invitation and subsequently a phone call to arrange the examination details. Among the 421 invited men, 243 agreed to participate, but 3 did not succeed in delivering a semen sample. Hence, the final F2 group comprised 240 men (57\% of all invited).

\section{Questionnaire}

On the day of attendance, the men returned a questionnaire they had received in advance. The questionnaire was based on that used for young Danish men ${ }^{12}{ }^{17}$ and included information on previous or current diseases, including any known history of fertility potential and some lifestyle factors like smoking and drinking habits.

\section{Semen samples}

Semen samples were produced by masturbation in a room close to the semen laboratory. The period of abstinence was recorded. The abstinence time was obtained differently in the two Faroese subgroups. In F1, the men were asked directly by the physician about the abstinence time while the F2 men themselves wrote down their abstinence time after they had some time to consider. The semen sample was analysed according to the WHO 1999 guidelines. ${ }^{18}$ Semen volume was estimated by weighing the collection tube with the semen sample and subtracting the weight of the empty preweighed tube, assuming that $1 \mathrm{ml}$ semen $=1 \mathrm{~g}$. For sperm motility assessment, $10 \mu \mathrm{l}$ of well-mixed semen was placed on a clean glass slide kept at $37^{\circ} \mathrm{C}$ and covered with a $22 \times 22 \mathrm{~mm}$ coverslip. The preparation was placed on the heated stage of a microscope at $37^{\circ} \mathrm{C}$ and immediately examined at $\times 400$ magnification. The sperm were classified as progressive motile (WHO class $\mathrm{AB}$ motility), locally motile (WHO class $\mathrm{C}$ motility) or immotile (WHO class D motility).

For the assessment of the sperm concentration, the samples were diluted in distilled water. The sperm concentration was subsequently assessed using a Bürker-Türk haemocytometer (Paul Marienfeld GmbH \& Co. KG, Lauda-Königshofen, Germany). However, a second delivery of Bürker-Türk chambers was $0.05 \mathrm{~mm}$ in depth rather than the standard $0.1 \mathrm{~mm}$ we had ordered. Because of that, 59 samples from F2 were analysed without knowing whether 0.05 or $0.1 \mathrm{~mm}$ deep counting chambers were used. As the semen samples had been stored in our bio-bank, the 59 samples were measured again with the $0.1 \mathrm{~mm}$ deep chamber to indicate if the original concentration assessment was correct (assuming that the $0.1 \mathrm{~mm}$ counting chamber was used originally) or should be doubled (assuming that the $0.05 \mathrm{~mm}$ counting chamber was used originally). Based on these reanalyses, the obtained original sperm concentrations were doubled for 23 samples (see online 
Table 1 Physical appearance and self-reported information of Faroese and Danish men

\begin{tabular}{|c|c|c|c|c|c|c|}
\hline & $\begin{array}{l}\text { Faroese men (F1) } \\
2007-2009(N=241)\end{array}$ & $\begin{array}{l}\text { Faroese men (F2) } \\
2009-2010(\mathrm{~N}=240)\end{array}$ & p Value* & $\begin{array}{l}\text { Faroese men (F1+F2) } \\
2007-2010(\mathrm{~N}=481)\end{array}$ & $\begin{array}{l}\text { Danish men (D) } \\
\text { 2006-2010 (N=1274) }\end{array}$ & p Value* \\
\hline \multicolumn{7}{|l|}{ Physical appearance } \\
\hline Age (years) & $25.3(24.2-26.7)$ & $23.0(22.0-24.0)$ & $<0.0005$ & $24.0(22.0-26.2)$ & $19.0(18.4-21.8)$ & $<0.0005$ \\
\hline Height (cm) & $180.0(169.9-189.9)$ & $180.0(170.0-193.0)$ & 0.1 & $180.0(170.0-191.0)$ & $181.6(171.1-193.0)$ & $<0.0005$ \\
\hline Weight (kg) & $80.3(64.2-106.2)$ & $78.00(62.9-105.1)$ & 0.2 & $80.0(63.1-105.9)$ & $74.1(60.1-96.0)$ & $<0.0005$ \\
\hline BMI $\left(\mathrm{kg} / \mathrm{m}^{2}\right)$ & $25.0(20.6-32.3)$ & $24.3(20.4-32.1)$ & 0.03 & $24.6(20.5-32.1)$ & $22.4(18.7-28.6)$ & $<0.0005$ \\
\hline Testes size $(\mathrm{ml}) \dagger$ & $21.5(15.3-25.0)$ & NA & & NA & $23(14-29)$ & \\
\hline \multicolumn{7}{|l|}{ Lifestyle } \\
\hline Alcohol per week (units) & $5(0-25)$ & $6(0-26)$ & 0.08 & $5(0-25)$ & $12(0-42)$ & $<0.0005$ \\
\hline Current smokers & 44.0 & 41.3 & 0.5 & 42.6 & 45.4 & 0.4 \\
\hline Mother smoked in pregnancy & $22.4 \ddagger$ & 37.5 & 0.05 & 33.6 & 29.1 & 0.4 \\
\hline Taken medication§ & 23.7 & 31.3 & 0.07 & 27.4 & 15.1 & $<0.0005$ \\
\hline \multicolumn{7}{|l|}{ Been treated forף } \\
\hline Cryptorchidism* & 3.3 & 7.5 & 0.04 & 5.4 & 5.0 & 0.8 \\
\hline \multicolumn{7}{|l|}{ Been diagnosed as havingt† } \\
\hline Cryptorchidism reported & 8.7 & 12.1 & 0.2 & 10.4 & 6.9 & 0.02 \\
\hline Hypospadias & 0.4 & 0.8 & 0.6 & 0.6 & 0.3 & 0.4 \\
\hline Sexually transmitted disease $\ddagger \ddagger$ & 9.1 & 10.8 & 0.5 & 10.0 & 6.2 & 0.007 \\
\hline Phimosis & 10.0 & 11.7 & 0.5 & 10.8 & 4.6 & $<0.0005$ \\
\hline Varicocele & 0.8 & 0.8 & 1.0 & 0.8 & 0.4 & 0.6 \\
\hline \multicolumn{7}{|l|}{ Have§§ } \\
\hline Ever caused a pregnancy $†$ & 25.7 & NA & & NA & 6.8 & - \\
\hline Fatherhood & 21.2 & 6.3 & $<0.0005$ & 13.7 & NA & - \\
\hline Experienced fertility problems $\uparrow \uparrow$ & 4.6 & 1.7 & 0.07 & 3.1 & 0.2 & $<0.0005$ \\
\hline
\end{tabular}

Results shown as medians (5-95th percentile) or percentages.

*Mann-Whitney test.

†Mean of two testes. Testes size was not measured in Faroese men born in 1986-1987, and the question 'Have you ever caused a pregnancy' was not asked in this group.

flnformation only available for 189 mothers.

$\S T a k e n$ any medication 3 months prior to participation in the study.

П'Have you ever been treated for...'

**Hormonal, surgical or combination.

††'Has a doctor ever diagnosed you as having....'

$\ddagger \ddagger$ Chlamydia, gonorrhoea, warts or herpes.

§\$ Have you ever...

११|Ever had regular intercourse without use of contraception for at least 6 months (Faroese men) or 1 year (Danish men) without partner becoming pregnant. BMI, body mass index; NA, not available. 
supplementary materials for details). To ascertain that the concentration could be replicated in thawed samples, samples known to be counted in $0.1 \mathrm{~mm}$ deep chamber were counted again. As anticipated, the results were replicated and shown to be accurate. From each semen sample, a smear for morphology evaluation was made, Papanicolaou stained and finally assessed according to 'strict criteria' at the Department of Growth and Reproduction (GR) at the National Hospital (Rigshospitalet, RH) in Denmark.

The Faroese semen analyses were performed by three technicians, who participated in a quality control testing course at RH and spent 2 weeks there to ensure comparable results. Furthermore, every 3 months, five blinded samples were sent from $\mathrm{RH}$, and sperm concentration results were compared with the results from their technicians. Throughout the study period, the variation was less than 10\% compared with the Danish technicians. Therefore, the methodological differences between the Faroese and the Danes should be considered very limited.

\section{Reproductive hormones}

On the day of the examination, a venous blood sample was drawn from each participant and centrifuged (3000 g, $10 \mathrm{~min})$. Serum was subsequently separated and kept frozen until it was analysed for reproductive hormones at GR at RH. Levels of follicle stimulating hormone (FSH), luteinising hormone (LH) and sex hormone-binding globulin (SHBG) were determined using a time-resolved fluoroimmunoassay (Delfia, Wallac, Turku, Finland). Testosterone (T) was determined using a COAT-A-COUNT solid-phase radioimmunoassay (RIA) (Siemens Medical Solutions, Malvern, Pennsylvania, USA) and oestradiol by RIA (Pantex, Santa Monica, California, USA). ${ }^{19}$ Inhibin B was analysed by a double antibody enzymeimmunometric assay using a monoclonal antibody raised against the inhibin $\beta B$-subunit in combination with a labelled antibody raised against the $\alpha$-subunit. Samples were analysed for inhibin B in 2009 using kit material from Oxford Bio-Innovation or in 2010 with kit material from DSL Beckman, USA. For all hormone assessments, internal standard samples were analysed and showed no need to correct for potential interassay variation, including the need for correction between the kit materials for inhibin B from the two suppliers. The free testosterone index (FT) was calculated from total testosterone and SHBG using a fixed albumin value according to Vermeulen et $a l^{20}$

\section{Physical examination}

Participants in the F1 subgroup underwent a physical examination performed by one of two examiners at the day of the semen sample delivery. Body weight and height were measured. The Tanner stage of pubic hair and genital development were recorded. Any abnormalities in the testis and penis, the possible presence of varicocele, hydrocele, hypospadias and testis tumour, the location of testis in scrotum and their consistency and that of epididymis were recorded. Testicular volumes were determined by use of a Prader orchidometer, and reported as the mean of two testes (table 1). Participants in the F2 study did not undergo a physical andrological examination.

\section{Comparison population: men from the general Danish population}

In Denmark, all young men, except those suffering from severe chronic diseases $(<15 \%)$, are required to attend a compulsory medical examination before they are considered for military service. ${ }^{5}$ Men are called upon at the age of 18-19 years, but some postpone this examination until completion of their education. Men attending the compulsory examination are invited to participate in a semen quality study, using the same basic study design including a physical examination as the Faroese, irrespective of whether they are declared fit for military service or not. For comparison with results from the Faroese studies, we utilised results for Danish men examined in 2006-2010, which has recently been published. ${ }^{12}$ The detailed description of the study population based on questionnaire information and results from the physical examination (see below) is summarised in table 1 .

\section{Comparison population: WHO reference group of fertile men}

The Faroese sperm count distribution was compared with the distribution for fertile men reported as the WHO reference group. ${ }^{13}$

\section{Statistics}

The crude means, medians, SDs, 5-95 percentiles and frequencies were used for basic descriptions of obtained results. The main outcome variables were semen volume, sperm concentration, total sperm count, percentage of motile spermatozoa, percentage of morphologically normal spermatozoa and serum level of the reproductive hormones. Differences in semen quality variables and reproductive hormone levels between groups were tested by linear regression adjusted for significant confounders. Semen volume, sperm concentration and total sperm count were best normalised by a cubic root transformation before regression analysis to correct for skewed distribution of residuals. The percentages of motile spermatozoa (ie, WHO class $\mathrm{AB}+\mathrm{C}$ ) were logit-transformed. Percentages of morphologically normal spermatozoa were close to normally distributed and entered the model untransformed. Reproductive hormone levels were natural logarithmic transformed. Correlations between reproductive hormone levels were assessed with Spearman correlation. Between-group differences for categorical variables were tested with nonparametric tests (Mann-Whitney). Total sperm count distribution differences between the Faroese men and the WHO reference distribution were tested by $\chi^{2}$ analyses. Association between year of birth and semen parameters was tested by linear regression. 
For F2, the abstinence time in hours showed a significant positive association to sperm concentration, semen volume and total sperm count. The effect was most pronounced for the period below $96 \mathrm{~h}$ and less above (for sperm concentration: $\beta$-value $=0.011, \mathrm{p}=0.01$ and $\beta$-value $=0.006, p=0.18$, respectively). The abstinence time was not significantly associated with sperm concentration in F1 ( $\beta$-value $=0.0001, \mathrm{p}=0.98$, and $\beta$-value $=0.0003, \mathrm{p}=0.5$, respectively) or total sperm count. Thus, abstinence time was entered as piecewise linear functions (linear splines); that is, one straight line for abstinence below 96 and another straight line for abstinence above 96 for semen volume, sperm concentration and total sperm counts. The duration from ejaculation to assessment was included as a confounder for sperm motility. No confounders were detected to affect morphology.

The following factors were evaluated as possible confounders for semen parameters and found to have no influence ( $p>0.05)$ : age, body mass index (BMI) (as continuous variable or categorised as $<18.49$; 18.5-25; $>25)$, smoking (yes/no) and season of year (spring (MarchMay), summer (June-August), autumn (SeptemberNovember) and winter (December-February)).

The same factors were evaluated as possible confounders for the reproductive hormones. Significant associations were observed for BMI and age but not for smoking. The effect of season was not systematic and thus not included as a confounder. Hour of day of blood sampling was included as a confounder for reproductive hormones, although it was only significantly associated with oestradiol, T and FT. p Values below $5 \%$ were considered to be statistically significant. Analyses were performed using PASW GradPack 19.0 (SPSS Inc, Chicago, Illinois, USA).

\section{RESULTS}

In table 1, physical appearance, lifestyle factors, reproductive health and self-reported information on fertility are displayed for the two subgroups of Faroese men, the combined Faroese group and the Danish comparison group. All men had their semen variables assessed. However, morphology scoring was missing for 50 Faroese men and 20 Danes, which is reported in table 2. Also, assessment of testis size was only performed on subgroup $\mathrm{F} 1$, which is stated in table 1 . Semen volume was not recorded for one Faroese man and motility measure was missing for one man.

The three groups differed in age with the F1 group being the oldest and the Danish group the youngest. Similarly, the F1 group had the highest BMI and the Danish group the lowest. Alcohol intake was lower among the Faroese men, and more Faroese men had caused a pregnancy/fathered a child.

\section{Semen variables}

Table 2 summarises the semen variables. Sperm concentrations for the Faroese men were lower than for the Danish men. However, semen volume was higher, and thus the

Table 2 Semen parameters in Faroese and Danish men

\begin{tabular}{|c|c|c|c|c|c|c|}
\hline & $\begin{array}{l}\text { Faroese } \\
\text { men }(\mathrm{F} 1) \\
2007-2009 \\
(\mathrm{~N}=241)\end{array}$ & $\begin{array}{l}\text { Faroese } \\
\text { men (F2) } \\
2009-2010 \\
(\mathrm{~N}=240)\end{array}$ & p Value & $\begin{array}{l}\text { Faroese men } \\
(\mathrm{F} 1+\mathrm{F} 2) \\
2007-2010 \\
(\mathrm{~N}=481)\end{array}$ & $\begin{array}{l}\text { Danish } \\
\text { men (D) } \\
2006-2010 \\
(\mathrm{~N}=1274)\end{array}$ & p Value \\
\hline \multicolumn{7}{|l|}{ Sperm concentration (mill/ml) } \\
\hline Mean (SD) & $54(56)$ & $60(60)$ & & $57(58)$ & $62(55)$ & \\
\hline Median (5-95 percentiles) & $38(1.6-156)$ & $41(2.2-182)$ & $0.5^{\star}$ & $40(1.9-174)$ & $48(4.0-169)$ & $<0.0005^{\star}$ \\
\hline \multicolumn{7}{|l|}{ Semen volume $(\mathrm{ml})$} \\
\hline Mean (SD) & $4.2(1.7)$ & $4.0(1.7)$ & & $4.1(1.7)$ & $3.6(3.1)$ & \\
\hline Median (5-95 percentiles) & $4.0(1.9-7.1)$ & $3.9(1.5-7.2)$ & $0.02^{*}$ & $3.9(1.8-7.1)$ & $3.3(1.3-6.3)$ & $<0.0005^{\star}$ \\
\hline \multicolumn{7}{|l|}{ Total sperm count (mill) } \\
\hline Mean (SD) & $214(205)$ & $215(206)$ & & $215(205)$ & $206(258)$ & \\
\hline Median (5-95 percentiles) & $154(3-586)$ & $159(9-638)$ & $0.8^{*}$ & $159(6-591)$ & $151(13-559)$ & $0.2^{*}$ \\
\hline \multicolumn{7}{|l|}{ Normal morphology (\%)†‡ } \\
\hline Mean (SD) & $7.5(4.3)$ & $6.3(4.5)$ & & $6.9(4.4)$ & $7.5(4.9)$ & \\
\hline Median (5-95 percentiles) & $7.5(1.0-15.0)$ & $5.0(0.5-15.0)$ & $<0.0005 \ddagger$ & $6.5(0.9-15.0)$ & $7.0(0.5-16.0)$ & $0.2 \S$ \\
\hline \multicolumn{7}{|l|}{ Motile sperm (\%)ף } \\
\hline Mean (SD) & $70(22)$ & $58(13)$ & & $64(19)$ & $65(16)$ & \\
\hline Median (5-95 percentiles) & $74(19-95)$ & $59(35-74)$ & $<0.0005^{\star *}$ & $64(24-90)$ & $68(33-85)$ & $0.02^{* \star}$ \\
\hline \multicolumn{7}{|l|}{ Period of abstinence (h) } \\
\hline Mean (SD) & $80(38)$ & $86(35)$ & & $83(37)$ & $77(96)$ & \\
\hline Median (5-95 percentiles) & $83(14-167)$ & $83(35-168)$ & $0.1 \S$ & $83(25-168)$ & $63(37-134)$ & $<0.0005 \S$ \\
\hline
\end{tabular}

${ }^{*}$ Regression analysis adjusting for duration of abstinence.

†Morphology was missing for 50 Faroese men $(\mathrm{F} 1+\mathrm{F} 2)$ and 20 Danish men.

$\ddagger$ Regression analysis without any adjustment.

§Mann-Whitney test.

TMotile sperm is the sum of progressively motile (WHO class $A B$ ) and local motile (WHO class $C$ ).

${ }^{* *}$ Regression analysis adjusting for delay from time of ejaculation to assessment of motility. 
total sperm counts did not differ. Similarly, overall motility and morphology did not differ between the Faroese and Danes, but it did differ between the two Faroese subgroups with higher values for the F1-subgroup. Figure 1A shows the distributions of total sperm counts of all the investigated Faroese men (blue bars) in categories defined from the reference levels according to centiles as described by the WHO (green bars). According to the figure, more Faroese men had lower sperm counts than the reference group $(\mathrm{p}<0.0001)$.

From the entire group of 481 men, $313(65 \%)$ were without any prior knowledge of andrological diseases or conditions that might be associated with risk of impaired testicular function (cryptorchidism, testicular torsion, orchitis, epididymitis, varicocele, hydrocele, prostatitis, hypospadias, phimosis, inguinal hernia, cystitis, sexually transmitted diseases, diabetes, thyroid diseases, experienced fertility problems). In this subgroup, 244 men (50\% of the entire group) had an ejaculation abstinence period of more than $48 \mathrm{~h}$. These 244 men also had
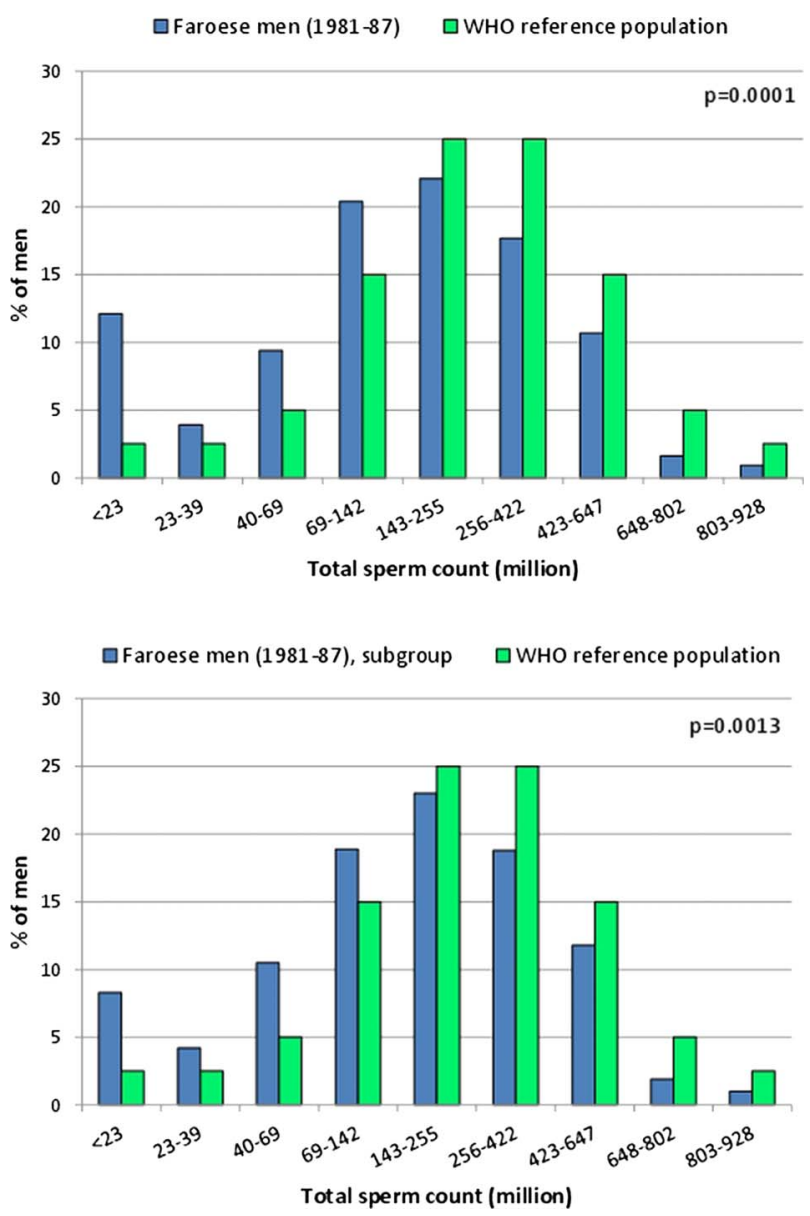

Figure 1 Distribution of total sperm count in Faroese men (blue bars) in categories defined from the reference levels according to centiles as described by WHO (green bars). In (A) all Faroese men are included. In (B) only men having an ejaculation abstinence period above $48 \mathrm{~h}$ and being 'without diseases' (see text for further explanation) are included. lower total sperm counts than men from the WHO reference group (figure $1 \mathrm{~B}$, lower panel, $\mathrm{p}=0.0013$ ).

The percentages of morphologically normal spermatozoa were inversely associated with year of birth, both for the entire group of Faroese men (figure 2A, trend $\mathrm{p}=0.002$ ) and for the subgroup of 244 men (figure $2 \mathrm{~B}$, trend $\mathrm{p}=0.018$ ). Morphology assessments were done in four different time periods, but the results did not differ according to these periods. Similar trends were seen when the estimates according to year of birth were adjusted for age, however, with broader CIs of the estimates. No other semen variables were associated with birth year and age at the time of investigation.

Approximately $14 \%$ of the participants were reported to have fathered a child. The confounder-adjusted estimates indicated higher sperm concentration, total sperm count, motility and morphologically normal spermatozoa in these men in comparison to non-fathers, all non-significant except for motility $(\mathrm{p}<0.0005)$. Semen volume was nonsignificantly lower in the father group $(p=0.7)$. When the
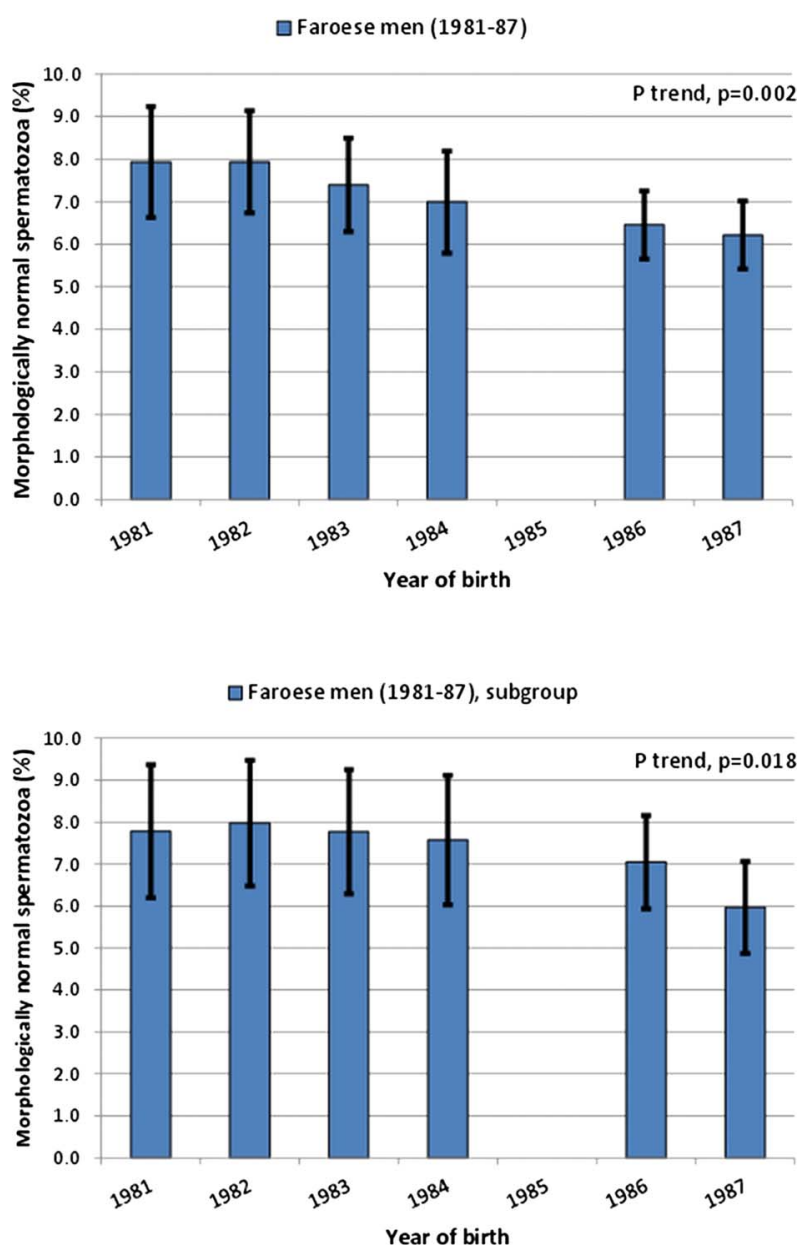

Figure 2 Percentage of morphologically normal spermatozoa according to year of birth. Whiskers show the $95 \%$ confidence interval of the estimated mean. In (A) all Faroese men are included. In (B) only men having an ejaculation abstinence period above $48 \mathrm{~h}$ and being 'without diseases' (see text for further explanation) are included. 
distribution of total sperm counts for these fathers were compared with the WHO reference group, no difference between the groups could be seen $(\mathrm{p}=0.1)$.

\section{Physical examination}

Only the men in the F1-group had a physical examination performed. Among these, $95 \%$ had a normal mean testicular volume (ie above $15 \mathrm{ml}$ ), which apparently was slightly lower than for the Danish men (table 1). A total of $2 \%$ did not have both testicles in scrotum, 8\% had soft testicles and $4 \%$ did not have adult pubic hair distribution (ie, Tanner stage 5 or 6 ). A varicocele was detected in $15 \%(5.8 \%$ grade $1,7.5 \%$ grade 2 and $1.7 \%$ grade 3 ) of the F1 cohort. In regression analyses, all semen variable estimates were higher in men without any varicocele, but they were all nonsignificant (all p>0.2). Hydrocele was detected in 5\%, and tended to be associated with a lower percentage of morphologically normal spermatozoa $(\mathrm{p}=0.05)$, but not with other semen variables (all $\mathrm{p}>0.6$ ).

\section{Smoking and BMI}

The men's tobacco smoking was non-significantly associated with lower semen volume, sperm concentration and total sperm counts (all $\mathrm{p}>0.1$ ) and higher percentage of normal forms and motile spermatozoa ( $\mathrm{p}=0.8$ and 0.2 , respectively). Maternal smoking during pregnancy was significantly associated with lower total sperm count (29\% reduction, 95\% CI 8\%; 45\%, $\mathrm{p}=0.02$ ), and non-significantly with semen volume $(9 \%$ reduction, $95 \%$ CI $-16 \%$; $2 \%, \mathrm{p}=0.05)$ and sperm concentration (22\% reduction, $95 \%$ CI $-40 \% ; 6 \%, \mathrm{p}=0.06$ ). There were no significant associations between maternal smoking, sperm motility and sperm morphology $(\mathrm{p}=0.5$ and 0.8 , respectively). BMI above 25 was not associated with adverse effects on any semen variable.

\section{Reproductive hormones}

Table 3 summarises the reproductive hormone levels for the two subgroups of Faroese men, the combined group and the Danish comparison group. Inhibin B tended to be slightly lower in the Faroese F1 group than in the F2 group, thus showing the same tendency as sperm concentration and total sperm count. FSH showed opposite directions resulting in lower inhibin $\mathrm{B} / \mathrm{FSH}$ ratio in the F1 group. In the combined Faroese group, both inhibin $\mathrm{B}$ and FSH individually were higher than in the Danish group, whereas the inhibin $\mathrm{B} / \mathrm{FSH}$ ratio was lower. In 2010 , the laboratory began to use a different kit material for the serum inhibin $B$ analyses. However, that the inhibin $\mathrm{B} / \mathrm{FSH}$ results did not differ according to this as is described in the Materials and methods section.

Although statistically significant, total testosterone (T) differed only slightly between the two Faroese groups and between the combined group and the Danes,. SHBG, by contrast, was considerably higher for the Faroese men and with no difference between the F1 and F2 men. Frequency analyses of the SHBG values divided into groups $(0-10,10-20$, etc.) showed that the higher SHBG values for the Faroese men were not caused by higher concentration in a specific subgroup but an overall shift to higher levels. Approximately $80 \%$ of the Faroese men had an SHBG concentration below $50 \mathrm{nmol} / 1$ while $80 \%$ of the Danish men had a concentration below $40 \mathrm{nmol} / \mathrm{l}$. The FT differed between the two Faroese groups, which combined, however, had nonsignificantly lower FT than the Danes. Oestradiol was higher in the F2 group than in the F1 group, thus leading to higher levels in the combined group of Faroese men than in the Danish men. The ratios T/LH, FT/LH and FT/oestradiol were all lower in the Faroese men than in the Danish men, whereas the estimated lower total $\mathrm{T}$ /oestradiol was non-significant.

SHBG and LH were positively correlated with $\mathrm{T}$ $(p<0.0005)$, whereas SHBG and LH did not correlate $(\mathrm{p}=0.1)$. FT was positively correlated with $\mathrm{LH}$ and $\mathrm{T}$ $(\mathrm{p}<0.0005)$, and negatively but not significantly correlated with SHBG $(\mathrm{p}=0.06)$

BMI was negatively associated with T, FT, SHBG, $\mathrm{T} / \mathrm{LH}, \mathrm{T} /$ oestradiol, FT/oestradiol and inhibin B $(\mathrm{p}<0.0005-0.042)$, and also negatively but nonsignificantly associated with LH $(p=0.3)$. Faroese smokers tended to have higher $\mathrm{T}$ than non-smokers ( $p=0.7$, adjusted for BMI effect), whereas the effect on the Danish men were highly significant $(p<0.0005)$. In the Faroese groups, LH, SHBG, oestradiol, FT/LH, FT/oestradiol and T/oestradiol tended to be higher in smokers, although all non-significantly. For the Danes, similar trends were detected, the effect on SHBG and LH being non-significant, whereas the remaining were significant with $\mathrm{p}$ values $<0.0005-0.05$. Further, maternal smoking during pregnancy for the Faroese men tended to be associated with lower LH and FT ( $\mathrm{p}=0.04$ and $0.02)$, whereas the effect on the remaining hormone levels were all highly non-significant $(\mathrm{p}=0.1-0.9)$. However, the difference (ie, significant levels in table 3) between the Faroese and Danish hormone levels did not change when including BMI or age as cofactors in the regression analyses, indicating that these factors cannot explain the difference in reproductive hormones between the Faroese and Danes.

\section{DISCUSSION}

This is the first study on testicular function to be conducted in the Faroe Islands. These young Faroese men had a lower sperm concentration but similar total sperm count compared to Danish men. Recent data have shown sperm counts to be low in young men from several European countries, however, slightly higher than among the Danes. ${ }^{5} 2122$ Thus, the semen quality of Danish men seems to be particularly low, and now we have shown the Faroese men to have a similar low level.

The low inhibin B/FSH ratio for the Faroese men corroborates the finding of low sperm counts, and provides independent evidence of poorer testicular function in 
Table 3 Reproductive hormones levels in Faroese and Danish men

\begin{tabular}{|c|c|c|c|c|c|c|}
\hline & $\begin{array}{l}\text { Faroese men (F1) } \\
2007-2009(N=241)\end{array}$ & $\begin{array}{l}\text { Faroese men (F2) } \\
2009-2010(\mathrm{~N}=240)\end{array}$ & p Value* & $\begin{array}{l}\text { Faroese men (F1+F2) } \\
2007-2010(\mathrm{~N}=481)\end{array}$ & $\begin{array}{l}\text { Danish men (D) } \\
\text { 2006-2010 (N=1274) }\end{array}$ & p Value ${ }^{*}$ \\
\hline \multicolumn{7}{|l|}{ FSH (IU/I) } \\
\hline Mean (SD) & $4.1(4.7)$ & $3.4(1.9)$ & & $3.8(3.6)$ & $2.8(1.8)$ & \\
\hline Median (5-95 percentile) & $3.1(1.3-8.5)$ & $2.9(1.1-7.0)$ & 0.05 & $3.1(1.2-7.7)$ & $2.5(1.0-6.0)$ & $<0.0005$ \\
\hline \multicolumn{7}{|l|}{ Inhibin B (pg/ml) } \\
\hline Mean (SD) & $196(79)$ & $220(84)$ & & $208(82)$ & $189(70)$ & \\
\hline Median (5-95 percentile) & $192(76-333)$ & $210(99-382)$ & 0.04 & $202(85-346)$ & $180(90-318)$ & 0.05 \\
\hline \multicolumn{7}{|l|}{ Inhibin B/FSH } \\
\hline Mean & $80(72)$ & $101(107)$ & & $91(92)$ & $100(89)$ & \\
\hline Median (5-95 percentile) & $57(11-203)$ & 70 (17-289) & 0.02 & $64(13-259)$ & $76(17-272)$ & $<0.0005$ \\
\hline \multicolumn{7}{|l|}{$\mathrm{LH}(\mathrm{IU} / \mathrm{I})$} \\
\hline Mean (SD) & $4.8(2.4)$ & $4.8(1.8)$ & & $4.8(2.1)$ & $3.5(1.5)$ & \\
\hline Median (5-95 percentile) & $4.5(2.1-8.5)$ & $4.6(2.1-7.9)$ & 1.0 & $4.5(2.1-7.9)$ & $3.3(1.6-6.3)$ & $<0.0005$ \\
\hline \multicolumn{7}{|l|}{ Testosterone $(\mathrm{nmol} / \mathrm{l})$} \\
\hline Mean (SD) & $21(7)$ & $23(7)$ & & $22(7)$ & $20(6)$ & \\
\hline Median (5-95 percentile) & $20(11-32)$ & $22(14-36)$ & 0.006 & $21(12-33)$ & $19(12-31)$ & $<0.0005$ \\
\hline \multicolumn{7}{|l|}{ Testosterone/LH } \\
\hline Mean & $5.0(2.3)$ & $5.9(7.2)$ & & $5.5(5.3)$ & $6.5(2.8)$ & \\
\hline Median (5-95 percentile) & $4.6(1.9-9.1)$ & $4.6(2.7-11.6)$ & 0.09 & $4.6(2.2-9.4)$ & $6.0(3.0-12.0)$ & $<0.0005$ \\
\hline \multicolumn{7}{|l|}{ Free testosterone $(\mathrm{pmol} / \mathrm{l})$} \\
\hline Mean & 409 (134) & $462(140)$ & & $439(134)$ & $454(142)$ & \\
\hline Median (5-95 percentile) & $397(211-633)$ & $442(262-707)$ & $<0.0005$ & $424(236-686)$ & $432(269-701)$ & 0.1 \\
\hline \multicolumn{7}{|l|}{ Free testosterone/LH } \\
\hline Mean & $99(44)$ & $125(175)$ & & $112(128)$ & $149(71)$ & \\
\hline Median (5-95 percentile) & $92(39-186)$ & $96(49-212)$ & 0.03 & $94(47-201)$ & $134(65-273)$ & $<0.0005$ \\
\hline \multicolumn{7}{|l|}{ Oestradiol (nmol/l) } \\
\hline Mean (SD) & $79(22)$ & $96(23)$ & & $88(24)$ & $80(24)$ & \\
\hline Median (5-95 percentile) & $78(46-113)$ & $94(62-140)$ & $<0.0005$ & $86(54-131)$ & $78(48-125)$ & $<0.0005$ \\
\hline \multicolumn{7}{|l|}{ Testosterone/oestradiol } \\
\hline Mean & $268(83)$ & $243(69)$ & & $255(78)$ & $258(70)$ & \\
\hline Median (5-95 percentile) & $262(140-409)$ & $236(146-375)$ & $<0.0005$ & $243(145-394)$ & $249(175-383)$ & 0.1 \\
\hline \multicolumn{7}{|l|}{ Free testosterone/oestradiol } \\
\hline Mean & $5332(1567)$ & $4955(1187)$ & & $5143(1400)$ & $5869(1768)$ & \\
\hline Median (5-95 percentile) & $5218(3230-7768)$ & $4876(3165-7201)$ & 0.002 & $5026(3182-7538)$ & $5636(3519-8956)$ & $<0.0005$ \\
\hline \multicolumn{7}{|l|}{ SHBG $(\mathrm{nmol} / \mathrm{l})$} \\
\hline Mean (SD) & $38(15)$ & $37(15)$ & & $38(15)$ & $30(12)$ & \\
\hline Median (5-95 percentile) & $36(17-62)$ & $35(19-68)$ & 0.7 & $36(18-65)$ & $29(14-51)$ & $<0.0005$ \\
\hline \multicolumn{7}{|l|}{ Time of blood sampling } \\
\hline Mean (SD) & $11: 56(2: 11)$ & $9: 57(1: 29)$ & & $10: 27(1: 56)$ & $10: 04(0: 42)$ & \\
\hline Median (5-95 percentile) & $10: 15(8: 40-15: 40)$ & $9: 37(8: 02-12.45)$ & $<0.0005 t$ & $10: 10(8: 30-15: 00)$ & $10: 00(9: 05-11: 20)$ & $0.6 \dagger$ \\
\hline
\end{tabular}


the Faroese men than in the Danes, although the medians were at a level where the association between sperm counts and inhibin B is weakened. ${ }^{23}$ When evaluating the results against the $\mathrm{WHO}$ reference population, Faroese men also had low sperm counts. The lower $\mathrm{T} /$ LH and FT/LH ratios point towards a lower Leydig cell capacity among Faroese men compared to Danes. Thus, the total testicular function among Faroese men may be at the same or lower level than Danes.

A greater proportion of men from the Faroese study populations had previously caused a pregnancy, which also would be expected since these men on average were almost 6 years older than the Danes. Furthermore, Faroese people are in general younger when parenting for the first time (25.5 years in 2011), ${ }^{24}$ and the fertility rate in the Faroe Islands is the highest in European countries $^{25}$ with 2.3 children per woman in $2011 .^{24}$ Traditionally, the family culture in the Faroe Islands is to have large families with many children; however, during the last 40 years, the fertility rate in the Faroe Islands has decreased from 3.4 children per woman in 1970 to 2.8 children per woman in 1990 to the current fertility rate of 2.3 children per woman in $2011 .^{24}$ Thus, the high birth rate most likely reflects socioeconomic factors rather than male fecundity. Despite this, more Faroese men from the study populations had experienced fertility problems than the Danish men. This is in agreement with the lower sperm concentration found in the Faroese men, but it should be taken into account that the Faroese men were asked about fertility problems on the basis of 6 months unprotected intercourse without achieving a pregnancy, and for the Danes it was based on 1 year. Therefore, the Danish men were more likely to have obtained a successful fertilisation having tried for twice as long. Furthermore, fewer Danish men may be aware of a fecundity problem because they were younger and had not yet tried to conceive.

The Faroese and Danish men were not exactly the same age. Semen quality has been reported to decline with increasing age. ${ }^{26-29}$ However, the Faroese studies and the comparative Danish studies were conducted at the same time periods, and the difference in age between groups is not likely to have influenced the semen parameters or the actual age of the participants which can still be regarded as young. Semen quality does on average not change between 19 and 23 years of age, and immaturity seems unlikely to explain the poor semen quality both among the Faroese and Danish men. ${ }^{30}$ Thus, age difference is not likely to explain the difference in semen quality.

The Faroese men were investigated during a relatively short time period and also represented narrow birth cohorts. Despite this, we actually detected an inverse association between the percentage of morphologically normal spermatozoa and the year of birth, a trend that was robust even when controlled for potential confounders. In a cohort like ours, the effect of birth year may be difficult to separate from the effect of age. However, when we modelled age as an explanatory factor and controlled for birth year, the trend effect of age was not apparent. Thus, our results suggest that younger men produced ejaculates with lower percentages of normal spermatozoa. This could be caused by certain unhealthy lifestyle factors among the younger cohorts, but not the older cohorts. Our currently existing data do not support that explanation, however, but the information we have could also be insufficient to exclude this suggestion. Alternatively, the birth cohort effect could reflect an increasing exposure during fetal life leading to impaired testicular development, which in adulthood would be reflected by reduced semen quality in line with the suggested Testicular Dysgenesis Syndrome hypothesis. ${ }^{31} 32$ The Faroese studies were investigated according to similar protocols and assessment of sperm concentration was controlled by the same quality control as the Danish study. The Faroese technicians were trained at $\mathrm{RH}$ in Denmark and the analyses used in both laboratories were identical, thus reducing the interobserver variation. Additionally, assessment of sperm morphologies was performed randomly by a single person. Frequency of motile sperm, however, is difficult to compare reliably between different groups. The motility assessment is highly subjective and controlling for quality control within this parameter is problematic. Previously, the interlaboratory variance of motility has shown to be of significant importance. ${ }^{33}$ Therefore, we hesitate to draw major conclusions regarding sperm motility.

The recording of abstinence time was obtained differently in the two Faroese subgroups. In the F1 group, the men were asked directly by the physician about the abstinence time while the F2 men themselves wrote down their abstinence time after they had some time to consider. This latter procedure is believed to be much more accurate, as the men were given time to reflect and were not expected to give an immediate verbal response. This assumption is supported by the fact that the anticipated relationship between abstinence time and sperm concentration only was clearly seen in the F2 group. Assuming that the reported abstinence time actually was longer among the Faroese men, these men still produced similar numbers of sperm as the Danish men, suggesting that the sperm production rate is lower in the Faroese population compared to Danes. This was, however, only confirmed for sperm concentration but not total sperm count when the abstinence period was accounted for in the statistical model.

As explained in the Materials and methods section, some of the semen samples were analysed with incorrect counting chambers. However, we corrected for this potential testing error. In addition, the statistical analyses performed with or without the 59 samples did not change the overall results, supporting the assumption that the corrections made were valid. A major advantage of the study was that the participants were not selected on fertility status. According to most of the Danish men, the main incentive for participation was the financial 
compensation. ${ }^{5}$ The F2 men were part of an existing birth cohort study, whereas F1 men were randomly recruited. Thus, we cannot exclude that men with suspicion of infertility may have been more interested in participating in the study. However, to control for this, we defined a subgroup of men with an abstinence period of more than $48 \mathrm{~h}$ and without any prior knowledge of andrological diseases and without known fertility, and still found that the same low sperm counts were detected. This problem with counting chambers, and the differences in semen quality and population characteristics between the two Faroese subpopulation exemplifies one of the main problems when comparing semen characteristics globally and over time, namely that standardised methods have to be applied and sufficiently large study populations investigated.

The finding of low semen quality and lower Leydig cell function in Faroese men cannot be explained by effects of confounders. Both high BMI and maternal smoking have previously been associated with reduced testicular function in some studies, but not all. ${ }^{34-40}$ In our cohort, the effects of these two usually quite robust confounders/ explanatory factors were not obvious on the semen parameters, neither on the reproductive hormones. However, our cohorts may have been too small to detect the effects of these two factors. Alternatively, other unidentified factors may have had a major influence masking the effect of BMI and maternal smoking on the semen parameters. The reason for the low testicular function of the Faroese young men is unknown, but this population is highly exposed to POPs derived solely from traditional marine food, which includes blubber from the pilot whale. Studies have shown associations between high polychlorinated biphenyl (PCB) levels and low semen quality, and since PCBs and p,p'-DDE have the potential to interfere with sex hormone functions, it is plausible to assume that these compounds can affect the function of these organs. ${ }^{41}$ There are some reports on the effect of POPs on male reproduction in humans, mainly indicating weak negative effects on sperm motility. ${ }^{41-43}$ For the two Faroese subgroups, we found that the percentage of motile cells was significantly lower compared with Danish men, indicating that increased exposure to endocrine disruptors can be one explanation.

Serum SHBG levels for the Faroese men were strikingly higher than in the Danes. SHBG is a sensitive marker for thyroid function. However, none of the study subjects were under treatment for hyperthyroidism. We did not measure thyroid hormones, but the men did not have any obvious clinical symptoms of hyperthyroidism. If a high thyroid level in general should explain the SHBG level, a significantly elevated LH and testosterone in men with low BMI would have been expected, which was not the case. Therefore, there is no indication that the high SHBG values are due to hyperthyroidism. Furthermore, alcohol consumption could not explain the high levels. One plausible explanation to the high SHBG levels could be the high PCB exposure mentioned above. A recent publication from the Faroe Islands found that SHBG increased at higher PCB exposure, both prenatally and currently. PCBs are known to affect a variety of liver functions, and it could be speculated that PCB-induced hepatic SHBG synthesis could play a possible role, although this possibility remains to be substantiated. ${ }^{44}$ We cannot elucidate further if PCB in our current study group can explain the SHBG levels.

It is known that the Faroese population differs genetically from other populations in many respects. There are several genetic diseases reported with very high frequency on the Faroese Islands. ${ }^{11}{ }^{45}$ However different genetic composition in populations may contribute to the explanation of differences in semen quality and would be worth further exploration.

In conclusion, we found that the semen quality among Faroese men is at the same low level as reported among Danish men. This low quality was corroborated by the reproductive hormone levels in Faroese men. The influence of environmental exposures and genetic factors on the semen quality has to be studied further.

\section{Author affiliations}

${ }^{1}$ Department of Occupational Medicine and Public Health, The Faroese Hospital System, Torshavn, Faroe Islands

${ }^{2}$ University Department of Growth and Reproduction, Rigshospitalet, Copenhagen, Denmark

${ }^{3}$ Department of Environmental Medicine, Institute of Public Health, Odense, Denmark

${ }^{4}$ Department of Environmental Health, Harvard School of Public Health, Boston, Massachusetts, USA

Acknowledgements The authors would like to thank Kaj Kallsberg, MD, for performing some of the clinical examinations and the laboratory technicians Maritu Arge Magnussen, Lindu Kannuberg and Annu Louisu Jacobsen for performing the semen analyses. Also, thanks to nurse Nanna Kallsberg for her hard work in recruiting the F1 subgroup.

Contributors PG, PW, NJ and TKJ made substantial contribution to conception and design. JH, MSP, PW participated in acquisition of data. All authors participated in interpretation of data. JH, MSP and NJ participated in drafting the article. All authors participated in revising the article critically for important intellectual content. All authors participated in final approval of the version to be published.

Funding This study has been supported economically by several grants: the Faroese Research Council, National Institute of Environmental Health Sciences, NIH (ES014460), European Commission through its Sixth Framework Programme for RTD (contract no. FO0D-CT-2006-016253, PHIME) and Kirsten and Freddy Johansens Fund (grant 95-103-72087).

\section{Competing interests None.}

Ethics approval The local Science Ethical Committee for the Faroe Islands and the Institutional Review Board at the Harvard School of Public Health have approved the study protocol, and all participants had given their informed consent.

Provenance and peer review Not commissioned; externally peer reviewed.

Data sharing statement No additional data are available.

\section{REFERENCES}

1. Carlsen E, Giwercman A, Keiding N, et al. Evidence for decreasing quality of semen during past 50 years. BMJ 1992;305:609-13.

2. Swan SH, Elkin EP, Fenster L. Have sperm densities declined? A reanalysis of global trend data. Environ Health Perspect 1997;105:1228-32. 
3. Swan SH, Elkin EP, Fenster L. The question of declining sperm density revisited: an analysis of 101 studies published 1934-1996. Environ Health Perspect 2000;108:961-6.

4. Jørgensen N, Andersen AG, Eustache F, et al. Regional differences in semen quality in Europe. Hum Reprod. 2001;16:1012-19.

5. Jørgensen N, Carlsen E, Nermoen I, et al. East-West gradient in semen quality in the Nordic-Baltic area: a study of men from the general population in Denmark, Norway, Estonia and Finland. Hum Reprod 2002;17:2199-208.

6. Bonde JP, Ernst E, Jensen TK, et al. Relation between semen quality and fertility: a population-based study of 430 first-pregnancy planners. Lancet 1998;352:1172-7.

7. Guzick DS, Overstreet JW, Factor-Litvak P, et al. Sperm morphology, motility, and concentration in fertile and infertile men. N Engl J Med 2001;345:1388-93.

8. Jedrzejczak P, Taszarek-Hauke G, Hauke J, et al. Prediction of spontaneous conception based on semen parameters. Int $J$ Androl 2008;31:499-507.

9. Gomendio M, Malo A, Garde J, et al. Sperm traits and male fertility in natural populations. Reproduction 2007:134:19-29.

10. Joensen UN, Jørgensen N, Rajpert-De Meyts E, et al. Testicular dysgenesis syndrome and Leydig cell function. Basic Clin Pharmacol Toxicol 2008;102:155-61.

11. Joensen F, Steuerwald EU, Rasmussen NH. Three congenital metabolic diseases in the Faeroe Islands. Incidence, clinical and molecular genetic characteristics of Faeroese children with glycogen storage disease type IIIA, carnitine transporter deficiency and holocarboxylase synthetase deficiency. Ugeskr Laeger 2006;168:667-70.

12. Jørgensen $\mathrm{N}$, Joensen UN, Jensen TK, et al. Human semen quality in the new millennium: a prospective cross-sectional population-based study of 4867 men. BMJ Open 2012;2:e000990. doi:10.1136/bmjopen-2012-000990

13. Cooper TG, Noonan E, von Eckardstein S, et al. World Health Organization reference values for human semen characteristics. Hum Reprod Update 2010;16:231-45.

14. Grandjean P, Weihe P, Jorgensen PJ, et al. Impact of maternal seafood diet on fetal exposure to mercury, selenium, and lead. Arch Environ Health 1992;47:185-95.

15. Grandjean P, Weihe P. Neurobehavioral effects of intrauterine mercury exposure: potential sources of bias. Environ Res 1993:61:176-83.

16. Grandjean P, Jorgensen PJ, Weihe P. Human milk as a source of methylmercury exposure in infants. Environ Health Perspect 1994;102:74-7.

17. Andersen AG, Jensen TK, Carlsen E, et al. High frequency of sub-optimal semen quality in an unselected population of young men. Hum Reprod 2000;15:366-72.

18. World Health Organization. Laboratory maual for examination of human semen and semen-cervicl mucus interaction. 4th edn. New York: Cambridge University Press, 1999.

19. http://hormlabvejl.regionh.dk/Metodeliste.asp

20. Vermeulen A, Verdonck L, Kaufman JM. A critical evaluation of simple methods for the estimation of free testosterone in serum. $J$ Clin Endocrinol Metab 1999;84:3666-72.

21. Punab M, Zilaitiene B, Jørgensen N, et al. Regional differences in semen qualities in the Baltic region. Int J Androl 2002;25:243-52.

22. Richthoff J, Rylander L, Hagmar L, et al. Higher sperm counts in Southern Sweden compared with Denmark. Hum Reprod 2002;17:2468-73.

23. Jørgensen N, Liu F, Andersson AM, et al. Serum inhibin-b in fertile men is strongly correlated with low but not high sperm counts: a coordinated study of 1,797 European and US men. Fertil Steril 2010;94:2128-34.

24. http://www.hagstovan.fo

25. http://www.cia.gov/library/publications/the-world-factbook/fields/2127. $\mathrm{html \# fo}$
26. $\mathrm{Li} \mathrm{Y}$, Lin H, Li Y, et al. Association between socio-psycho-behavioral factors and male semen quality: systematic review and meta-analyses. Fertil Steril 2011;95:116-23.

27. Auger J, Kunstmann JM, Czyglik F, et al. Decline in semen quality among fertile men in Paris during the past 20 years. N Engl $J$ Med 1995;332:281-5.

28. Irvine S, Cawood E, Richardson D, et al. Evidence of deteriorating semen quality in the United Kingdom: birth cohort study in 577 men in Scotland over 11 years. BMJ 1996;312:467-71.

29. Jensen TK, Keiding N, Scheike T, et al. Declining human fertility? Fertil Steril 2000;73:421-3.

30. Carlsen E, Swan SH, Petersen JH, et al. Longitudinal changes in semen parameters in young Danish men from the Copenhagen area. Hum Reprod 2005;20:942-9.

31. Skakkebaek NE, Rajpert-De Meyts E, Main KM. Testicular dysgenesis syndrome: an increasingly common developmental disorder with environmental aspects. Hum Reprod 2001:16:972-8.

32. Jørgensen N, Meyts ER, Main KM, et al. Testicular dysgenesis syndrome comprises some but not all cases of hypospadias and impaired spermatogenesis. Int $J$ Androl 2010;33:298-303.

33. Jørgensen N, Auger J, Giwercman A, et al. Semen analysis performed by different laboratory teams: an intervariation study. Int $J$ Androl 1997:20:201-8.

34. Povey AC, Clyma JA, McNamee R, et al. Modifiable and non-modifiable risk factors for poor semen quality: a case-referent study. Hum Reprod 2012;27:2799-806.

35. MacDonald AA, Herbison GP, Showell M, et al. The impact of body mass index on semen parameters and reproductive hormones in human males: a systematic review with meta-analysis. Hum Reprod Update 2010;16:293-311.

36. Jensen TK, Jørgensen N, Punab M, et al. Association of in utero exposure to maternal smoking with reduced semen quality and testis size in adulthood; a cross sectional study of 1770 young men from the general population in five European countries. Am J Epidemiol 2004;159:49-58.

37. Jensen TK, Andersson AM, Jørgensen N, et al. Body mass index in relation to semen quality and reproductive hormones among 1558 Danish men. Fertil Steril 2004;82:863-70.

38. Paasch U, Salzbrunn A, Glander HJ, et al. Semen quality in sub-fertile range for a significant proportion of young men from the general German population: a co-ordinated, controlled study of 791 men from Hamburg and Leipzig. Int J Androl 2008;31:93-102.

39. Ravnborg T, Jensen TK, Andersson A-M, et al. Prenatal and adult exposures to smoking are associated with adverse effects on reproductive hormones, semen quality, final height and body mass index. Hum Reprod 2011;26:1000-11.

40. Virtanen HE, Sadov S, Toppari J. Prenatal exposure to smoking and male reproductive health. Curr Opin Endocrinol Diabetes Obes 2012;19:228-32.

41. Elzanaty S, Rignell-Hydbom A, Jönsson BA, et al. Association between exposure to persistent organohalogen pollutants and epididymal and accessory sex gland function: multicentre study in Inuit and European populations. Reprod Toxicol 2006;22:765-73.

42. Hauser R, Altshul L, Chen Z, et al. Environmental organochlorines and semen quality: results of a pilot study. Environ Health Perspect 2002;110:229-33.

43. Richthoff J, Rylander L, Jönsson BA, et al. Serum levels of $2,2^{\prime}, 4,4^{\prime}, 5,5^{\prime}$-hexachlorobiphenyl (CB-153) in relation to markers of reproductive function in young males from the general Swedish population. Environ Health Perspect 2003;111:409-13.

44. Grandjean P, Grønlund C, Kjær IM, et al. Reproductive hormone profile and pubertal development in 14-year-old boys prenatally exposed to polychlorinated biphenyls. Reprod Toxicol 2012;34:498-503.

45. Milman N, Steig T, Koefoed $\mathrm{P}$, et al. Frequency of the hemochromatosis HFE metations C282Y, H63D, and S65C in blood donors in the Faroe Islands. Ann Hematol 2004;84:146-9. 